\title{
Serum biomarker screening for the diagnosis of early gastric cancer using SELDI-TOF-MS
}

\author{
PING LI $^{1}$, DIANLIANG ZHANG ${ }^{2}$ and CHUNBAO GUO ${ }^{3}$ \\ Departments of ${ }^{1}$ Ultrasound, and ${ }^{2}$ General Surgery, The Affiliated Hospital of Medical College, Qingdao University, Qingdao; \\ ${ }^{3}$ Department of Hepatobiliary Surgery, Children's Hospital of Chongqing Medical University, Chongqing, P.R. China
}

Received December 4, 2011; Accepted March 3, 2012

DOI: $10.3892 / \mathrm{mmr} .2012 .834$

\begin{abstract}
In this study, we performed a proteomic analysis of sera from stage I gastric cancer patients using surfaceenhanced laser desorption/ionization time-of-flight mass spectrometry (SELDI-TOF-MS) and established a diagnostic model for the early diagnosis of stage I gastric cancer. Serum samples from 169 gastric cancer patients and 83 age- and gender-matched healthy individuals were analyzed by SELDITOF-MS ProteinChip array technology. The SELDI-TOF-MS spectral data were analyzed using the Biomarker Wizard ${ }^{\mathrm{TM}}$ and Biomarker Patterns ${ }^{\mathrm{TM}}$ software to find differential proteins and develop a classification tree for gastric cancer. A total of 34 mass peaks were identified. Six peaks at a mass-to-charge ratio $(\mathrm{m} / \mathrm{z})$ of $2873,3163,4526,5762,6121$ and 7778 were used to construct the diagnostic model. The model effectively distinguished gastric cancer samples from control samples, achieving a sensitivity and specificity of 93.49 and $91.57 \%$, respectively. In addition, we identified 3 of the 6 protein peaks at 2873,6121 and $7778 \mathrm{~m} / \mathrm{z}$, which distinguished between stage I and stage II/III/IV gastric cancer. The model had an accuracy of $88.89 \%$ for the identification of stage I gastric cancer. In conclusion, the diagnostic model for the detection of serum proteins by SELDI-TOF-MS ProteinChip array technology correctly distinguishes gastric cancer from healthy samples, and has the ability to screen and distinguish between early gastric cancer from advanced gastric cancer.
\end{abstract}

Correspondence to: Dr Dianliang Zhang, Department of General Surgery, The Affiliated Hospital of Medical College, Qingdao University, No. 16 Jiangsu Rd., Qingdao 266003, P.R. China E-mail:phdzdl@yahoo.com

Dr Chunbao Guo, Department of Hepatobiliary Surgery, Children's Hospital of Chongqing Medical University, No. 136 Zhongshan 2 Rd., Chongqing 400014, P.R. China

E-mail: gchunbao@yahoo.com.cn

Key words: gastric cancer, biomarkers, surface-enhanced laser desorption/ionization time-of-flight mass spectrometry, serum proteomic patterns

\section{Introduction}

Gastric cancer is the fourth most common malignancy and second leading cause of cancer-related mortality worldwide (1). Almost two-thirds of gastric cancer cases and deaths occur in less developed regions, including China (2). Although with the development and refinement of radiographic upper gastrointestinal examination and endoscopy, neoadjuvant treatment and surgical treatment have undergone significant changes in the past decades, the prognosis for gastric cancer patients remains poor. Its prognosis is determined by clinical stage. According to a previous study, the estimated adjusted survival 5 years after surgery is $82.9 \%$ for stage I, $62.8 \%$ for stage II, $17.8 \%$ for stage III and $3.3 \%$ for stage IV patients (3). Overall 5 -year survivals of approximately $20 \%$ or less are frequently reported.

The early diagnosis and early treatment of gastric cancer patients is the key to improving prognosis. When gastric cancers confined to the mucosa or submucosa are identified at an early stage, the 5-year survival is $90 \%$ or more (3-5). Thus, the identification of early-stage disease may be the most promising method to reduce gastric cancer mortality. Currently, endoscopic biopsy and histopathological evaluation of tumor resection margins are considered as a gold standard in the diagnosis of gastric cancer. However, this technique has evident disadvantages, in that it is invasive, time-consuming and expensive. More importantly, many patients are diagnosed with gastric cancer when metastasis has already occurred, thus limiting treatment efficacy. Additionally, the considerable expense of endoscopic biopsy or histopathological evaluation of tumor resection margins weakens the cost-effectiveness when screening programs for large populations are developed. Therefore, it is necessary to develop molecular biological techniques which are less invasive and more sensitive to improve the early diagnostics of gastric cancer. The identification of tumor biomarkers with high specificity and sensitivity would be desirable for the screening and diagnosis of early gastric cancer.

Biomarkers are defined as biological variables that correlate with biological outcome, and cancer biomarker discovery strategies that target expressed proteins are becoming increasingly popular (7). A great deal of effort has been spent in the search of tumor biomarkers, in order to improve the understanding of the behavior of gastric cancer and to identify 
biomarkers that would improve cure rates by early detection and diagnosis. To date, a number of widely used biomarkers for gastric cancer have been identified, such as carcinoembryonic antigen (CA) 19-9, CA 50 and CA 72-4 (8-10). However, the sensitivity and specificity of these biomarkers are not sufficient to detect early-stage gastric cancer. There are also disadvantages in using a single biomarker, such as weak specificity and a low positive rate. Few studies have simultaneously evaluated more than one candidate biomarker to enhance the diagnostic sensitivity and specificity. At the same time, these studies have led to the belief that no single biomarker is likely to prove sufficiently predictive $(11,12)$. Therefore, a logical development to improve the early diagnosis of cancer is to simultaneously screen for multiple biomarkers to increase the probability of detection (13-15).

Surface-enhanced laser desorption/ionization time-offlight mass spectrometry (SELDI-TOF-MS) ProteinChip is an innovative proteomic technology that enables the highthroughput analysis of a variety of serum samples from patients with gastric cancer and healthy controls, for the discovery of multiple specific protein biomarkers that could be used for the early diagnosis of gastric cancer. It has the advantages of being simple to operate, it is fast, and provides high throughput screening, high sensitivity and specificity. Over the years, SELDI-TOF-MS has been successfully applied in many types of tumors. A number of biomarkers in this process have been identified and further characterized, associated with cancers such as breast (16-18), liver (19-21), lung $(22,23)$, prostate (24) and ovarian cancers (25). These studies suggest that SELDITOF-MS ProteinChip technology can distinguish cancer patients from normal subjects with relatively high sensitivity and specificity. Thus, SELDI proteomic analysis is a valuable method to detect biomarkers or profile biomarkers within different sample groups; for example healthy individuals and cancer patients (26). However, at present, there are no satisfactory diagnostic biomarkers for early gastric cancer. The purpose of our study was to perform a proteomic analysis of sera from gastric cancer patients using SELDI-TOF-MS, and establish a useful diagnostic model for identifying gastric cancer or early gastric cancer.

\section{Materials and methods}

Study population. Consecutive patients with primary gastric cancer were prospectively considered from September 1, 2009 to September 1, 2010 at the Affiliated Hospital of Medical College, Qingdao University. All of the patients were diagnosed by histological examination. Patients who had received prior treatment before admission were excluded. To be eligible for enrollment, the subjects had to belong to the Chinese Han population. The protocol was approved by the Ethics Committee of Qingdao University, and informed consent was obtained from each patient or a close relative. Tumor TNM staging was recorded according to the classification of the American Joint Committee on Cancer (7th ed., 2010). We also excluded all of the volunteers and gastric cancer patients with infectious diseases, such as acute inflammation, viral HIV, HBV and $\mathrm{HCV}$ infections, as well as other serious diseases. The controls were recruited from hospital attendees in 2 centers with no family history of gastric cancer, and were followed-up for up to a maximum of 5 years, in which none developed gastric cancer. They were matched with patients for age and gender, without any malignant diseases and infectious disorders.

Serum samples. Immediately after admission and prior to any surgical or medical procedure, $5 \mathrm{ml}$ of peripheral blood samples were collected from patients on an empty stomach early in the morning, and placed in glass tubes without additive (BD Vacutainer ${ }^{\mathrm{TM}}$; BD Vacutainer Systems, Franklin Lakes, NJ, USA). The blood was centrifuged at 2,000 rpm for $10 \mathrm{~min}$ within $4 \mathrm{~h}$ after collection, and then stored at $-80^{\circ} \mathrm{C}$ until detection.

ProteinChip analysis. An aliquot of the stored sera was used for the SELDI-TOF-MS analysis. The SELDI-TOF-MS technology (Ciphergen Biosystems, Fremont, CA, USA) consists of 3 major components: the ProteinChip array, the reader and the software. The ProteinChip array is a $10-\mathrm{mm}$ wide 680 -mm long chip with 8 2-mm spots comprised of a specific chromatographic surface. Each surface is designed to select proteins from crude extracts according to general or specific protein properties. Each spot contains either a chemically- (anionic, cationic, hydrophobic or metal) or biochemically-treated surface. In our experiments, a cationic exchanger (WCX2) was used. In brief, $10 \mu \mathrm{l}$ of each serum sample and $90 \mu \mathrm{l}$ of a solution containing 0.5\% CHAPS (Sigma Inc., St. Louis, MO, USA) in phosphate-buffered saline (pH 7.4) were added to each well of a 96-well plate. The mixture was vortex-mixed at $4^{\circ} \mathrm{C}$ for $15 \mathrm{~min}$, followed by the addition of $100 \mu 1$ of Cibacron Blue 3GA (Sigma; prepared and balanced in $0.5 \%$ CHAPS 3 times). The plates were placed on a platform shaker at $4^{\circ} \mathrm{C}$ for $60 \mathrm{~min}$. After centrifugation, the supernatant (40 $\mu \mathrm{l}$ ) was transferred onto the WCX2 chips, so that each chip (8-spot format) held 4 tumorous and 4 healthy samples to rule out systematic error. All samples, including the training set, test set and normal serum quality control (QC) sample, were positioned randomly on the chips. The chips were placed in a bioprocessor (Ciphergen Biosystems Inc.), which holds 12 chips and allows a larger volume of serum to be applied to each chip array. The samples were allowed to react with the surface of the WCX 2 chip for $60 \mathrm{~min}$ at room temperature. The chips were then washed 3 times by gentle agitation on a platform shaker at a speed of $700 \mathrm{rpm}$ for $5 \mathrm{~min}$ with $200 \mu \mathrm{l}$ of $20 \mathrm{mmol} / \mathrm{l} \mathrm{HEPES}$ (pH 7.4), air dried and crystallized by the addition of $\alpha$-cyano4-hydroxycinnamic acid (CHCA; Ciphergen Biosystems Inc.). The chips were read on a protein biological system II (PBS-II) mass spectrometer reader (Ciphergen Biosystems Inc.). All spectra were compiled, and qualified mass peaks (signal-tonoise ratio $>5$ ) with mass-to-charge ratios ( $\mathrm{m} / \mathrm{z}$ ) between 2,000 and 30,000 were autodetected. Peak clusters were completed using second pass peak selection (signal-to-noise ratio $>2$, within a $0.3 \%$ mass window), and estimated peaks were added. The relative peak intensities, normalized to a total ion current of $\mathrm{m} / \mathrm{z}$ between 2,000 and 30,000, were expressed as arbitrary units. All these were performed using ProteinChip Software 3.0.2 (Ciphergen).

Statistical analysis. Peak intensities were normalized by total ion currency and analyzed by Biomarker Wizard software (Ciphergen Biosystems Inc.) to identify the peaks showing significantly different intensities between normal and cancer 
Table I. General information of the gastric cancer patients and control groups.

\begin{tabular}{lccccc}
\hline $\begin{array}{l}\text { Histological } \\
\text { classification }\end{array}$ & Samples & Male samples & Female samples & $\begin{array}{c}\text { Age range } \\
\text { (years) }\end{array}$ & $\begin{array}{c}\text { Mean age } \\
\text { (years) }\end{array}$ \\
\hline Normal & 83 & 56 & 27 & $33-80$ & 60.3 \\
Gastric cancer & & & & & \\
Stage I & 27 & 18 & 9 & $45-78$ & 63.5 \\
Stage II & 45 & 32 & 13 & $36-79$ & 58.7 \\
Stage III & 56 & 28 & 19 & $34-85$ & 62.3 \\
Stage IV & 37 & & & & 54.5 \\
\hline
\end{tabular}

groups. The Mann-Whitney U test was used for statistical analysis of differences between the cancer group and the control group. Classification analysis and construction of decision trees were performed with the Biomarker Patterns software 5.0 (Ciphergen Biosystems Inc.). A discriminatory pattern that distinguished normal from gastric cancer samples was developed from a training set of mass spectra; this diagnostic pattern was then applied to a blinded set of samples from cancer patients and healthy subjects.

\section{Results}

A total of 252 serum samples, including 169 pathologically confirmed gastric cancer patients (group 1 included 27 stage I, 45 stage II, 56 stage III and 37 stage IV patients) and 83 healthy subjects (group 2) were collected (Table I). The reproducibility of the ProteinChip SELDI assays using the pooled sera from 83 control samples was determined. Four chip chemistries [hydrophobic surface, immobilized metal affinity capture, weak cation exchange (WCX-2) and strong anion exchange] were evaluated to investigate which provided the best serum profile. Our determinations revealed that the WCX-2 chip provided the most discriminating pattern for constructing a decision tree (Table II). The peaks were analyzed in the mass rang of 2,000 to $30,000 \mathrm{~m} / \mathrm{z}$ and a total of 34 mass peaks were identified.

Biomarker characteristics of serum from gastric cancer patients and normal volunteers. There were statistical differences between 6 protein peaks located at 2873, 4526, 3163 , 5762,6121 and $7778 \mathrm{~m} / \mathrm{z}(\mathrm{P}<0.05)$; the intensity of protein peaks at $2873 \mathrm{~m} / \mathrm{z}$ in the sera from patients with gastric cancer was clearly higher than that of the healthy controls $(\mathrm{P}<0.05)$. Bi-peak and tri-peaks (5762 and $3163 \mathrm{~m} / \mathrm{z}$ ) were also observed in the sera from patients with gastric cancer. Furthermore, the protein peaks at 7778,4526 and $6121 \mathrm{~m} / \mathrm{z}$ in the sera from patients with gastric cancer were down-regulated compared to normal healthy volunteers (Table III). Using the above profiles, 158 of the 169 patients diagnosed pathologically with gastric cancer were correctly identified by SELDI. Seventy-six of the 83 healthy volunteers were correctly identified as normal. The sensitivity of gastric cancer identification was $93.49 \%$ $(158 / 169)$ for patients, whereas the specificity of control verification was $91.57 \%(76 / 83)$.

Biomarker characteristics of serum from stage I and stage II/ III/IV gastric cancer patients. In the mass spectral patterns
Table II. Performance of the decision tree analysis of gastric cancer in the training set.

Gold standard Gold standard Total (D+)

(D-)

\begin{tabular}{lrrr}
\hline Training set (T+) & 158 & 7 & 165 \\
Training set (T-) & 11 & 76 & 87 \\
Total & 169 & 83 & 252 \\
\hline
\end{tabular}

Table III. Average peak intensity of 6 distinct protein spectra found in the sera of patients with gastric cancer compared to healthy volunteers (mean $\pm \mathrm{SD})$.

\begin{tabular}{lrcr}
\hline $\mathrm{m} / \mathrm{z}$ & $\begin{array}{c}\text { Healthy } \\
\text { volunteers }\end{array}$ & $\begin{array}{c}\text { Stage I } \\
\text { gastric cancer }\end{array}$ & $\begin{array}{r}\text { Stage II/III/IV } \\
\text { gastric cancer }\end{array}$ \\
\hline $2873 \uparrow$ & $1.02 \pm 0.40$ & $2.13 \pm 1.12$ & $6.00 \pm 3.36$ \\
$3163 \uparrow$ & $0.90 \pm 0.60$ & $1.78 \pm 0.66$ & $4.81 \pm 2.38$ \\
$4526 \downarrow$ & $5.13 \pm 3.06$ & $2.57 \pm 0.57$ & $0.52 \pm 0.40$ \\
$5762 \uparrow$ & $3.67 \pm 2.77$ & $8.09 \pm 4.40$ & $12.70 \pm 3.70$ \\
$6121 \downarrow$ & $15.90 \pm 5.00$ & $9.70 \pm 6.90$ & $3.00 \pm 1.40$ \\
$7778 \downarrow$ & $17.11 \pm 0.42$ & $8.36 \pm 4.15$ & $3.05 \pm 2.10$
\end{tabular}

$\uparrow$ and $\downarrow$ represent up- and down-regulated expression in gastric cancer, respectively.

identified above, we observed that 3 protein peaks (2873, 5762 and $7778 \mathrm{~m} / \mathrm{z}$ ) were differentially expressed in early gastric cancer (stage I) compared to stage II, III and IV cases. Using these criteria, stage I gastric cancer was correctly identified in 24 out of 27 samples. Therefore, SELDI-TOF-MS can distinguish between stage I and stage II/III/IV gastric cancer. The biomarkers had an accuracy of $88.89 \%$ for the identification of stage I gastric cancer.

\section{Discussion}

Early diagnosis improves the long-term survival chances of patients with stomach cancers, and currently no satisfactory biomarkers for the diagnosis of early gastric cancer exist. It is very important to develop a convenient and non-invasive 
diagnostic method for routine screening and thereby increase the early diagnosis of cancers, which may lead to more patients being cured and reduced mortality. However, there are several obstacles in identifying serum biomarkers for cancer. Many potentially valuable biomarkers are expressed at very low levels and are difficult to detect. In addition, protein concentrations are unstable and may change with stress, disease or treatment. Proteins can be modified by cleavage or by the addition of new functional groups.

In our study, we analyzed protein expression patterns in sera obtained from gastric cancer patients and normal controls using the SELDI ProteinChip array, and constructed 2 decision trees for differentiating gastric cancer patients from normal individuals. Six peaks of $2873,4526,3163,5762,6121$ and $7778 \mathrm{~m} / \mathrm{z}$ were used to discriminate the 2 groups; of note, 3 peaks, 2873, 6121 and $7778 \mathrm{~m} / \mathrm{z}$, were differentially expressed in stage I gastric cancer compared to stage II, III and IV cases. The 2 decision trees constructed using those peaks showed high sensitivity and specificity in discriminating stage I gastric cancer, stage II/III/IV gastric cancer patients and controls.

Notably, several studies have addressed serum-based molecular markers, but each study showed different mass peaks and divergent results. Lu et al analyzed 65 serum samples from gastric cancer patients (27). Five protein peaks at 2046, 3179, 1817, 1725 and $1929 \mathrm{~m} / \mathrm{z}$ were components of the biomarker pattern for the diagnosis of gastric cancer, and 1 protein peak (4665 m/z) distinguished between stage I/II and stage III/IV with a specificity and sensitivity of $91.6 \%$ (11/12) and $95.4 \%$ $(21 / 22)$, respectively. The detection range of the mass peak (1,500-20,000 Da) was different from ours (2,000-30,000 Da). This may have caused the disparity between the results of our 2 studies.

Another decision tree analysis of serum proteomic patterns was performed by Anderson et al (28). They showed that 8 of 9 stage I gastric cancers $(88.9 \%$ sensitivity for stage I) were correctly classified. It is very important study for the detection of stage I gastric cancers by SELDI-TOF-MS, but they studied few cases. Ebert et al (29) constructed a decision tree by analysis serum proteomic patterns. Their system was capable of differentiating the gastric cancer samples from the others with a specificity of $88.0 \%$ and a sensitivity of $85.3 \%$. Unlike the present study, Su et al used SAX2 (strong anionic exchanger) chips. Liang et al showed that the comparison of protein expression profiles from serum appears to provide an effective approach to identify unique biomarkers for gastric cancer and gastritis, but they did not construct model trees using mass peaks (30).

Our findings are in general agreement with those reported by previous authors, thus providing additional confirmation that a proteomic approach accurately identifies gastric cancer patients from healthy controls. We also show a potential advantage of SELDI-TOF-MS, which is the ability to detect stage I gastric cancer. This may have significant implications for its utility in screening for early gastric cancer. Although the limitations of SELDI-TOF-MS study design and its analysis have been discussed in some detail in the literature (31), the potential implications of such a proteomic spectrum analysis for the identification of novel tumor biomarkers are huge.

In conclusion, we set up 2 serum proteomic patterns by SELDI-TOF-MS that have potential for clinical use. This analysis distinguishes gastric cancer patients from healthy controls, and has the ability to screen and distinguish between early gastric cancer from advanced gastric cancer. Using the diagnostic patterns to diagnose early gastric cancer can obtain a higher positive rate, higher sensitivity and specificity. Therefore, it seems desirable to know the identity of the biomarkers in the pattern in order to understand their significance in gastric cancer pathogenesis, staging and prognosis. We hope in the future to identify these protein peaks and combine them with other markers, such as CEA or CA199 or CA125, to assess therapeutic response and increase early detection of early gastric cancer.

\section{Acknowledgements}

This study was supported by the Shandong Scientific and Technological Project (2008GG30002034).

\section{References}

1. Yang L, Parkin DM, Ferlay J, Li L and Chen Y: Estimates of cancer incidence in China for 2000 and projections for 2005. Cancer Epidemiol Biomarkers Prev 14: 243-250, 2005.

2. Yang L: Incidence and mortality of gastric cancer in China. World J Gastroenterol 12: 17-20, 2006.

3. Spataro V, Genoni M, Maurer C and Muller W: Stomach cancer: 10 years' experience with surgical treatment and possibilities for improving the prognosis. Helv Chir Acta 59: 589-595, 1993.

4. Cenitagoya GF, Bergh CK and Klinger-Roitman J: A prospective study of gastric cancer. 'Real' 5-year survival rates and mortality rates in a country with high incidence. Dig Surg 15: 317-322, 1998.

5. Maruyama K, Okabayashi K and Kinoshita T: Progress in gastric cancer surgery in Japan and its limits of radicality. World J Surg 11: 418-425, 1987.

6. Bunt AM, Hermans J, Smit VT, van de Velde CJ, Fleuren GJ and Bruijn JA: Surgical/pathologic-stage migration confounds comparisons of gastric cancer survival rates between Japan and Western countries. J Clin Oncol 13: 19-25, 1995.

7. Jankowski JA and Odze RD: Biomarkers in gastroenterology: between hope and hype comes histopathology. Am J Gastroenterol 104: 1093-1096, 2009.

8. Qiu MZ, Lin JZ, Wang ZQ, et al: Cutoff value of carcinoembryonic antigen and carbohydrate antigen 19-9 elevation levels for monitoring recurrence in patients with resectable gastric adenocarcinoma. Int J Biol Markers 24: 258-264, 2009.

9. Marrelli D, Roviello F, De Stefano A, et al: Prognostic significance of CEA, CA 19-9 and CA 72-4 preoperative serum levels in gastric carcinoma. Oncology 57: 55-62, 1999.

10. Carpelan-Holmstorm M, Louhimo J, Stenman UH, Alfthan $\mathrm{H}$ and Haglund C: CEA, CA 19-9 and CA72-4 improve the diagnostic accuracy in gastrointestinal cancers. Anticancer Res 22: 2311-2316, 2002.

11. Adam B-L, Vlahou A, Semmes OJ and Wright GL Jr: Proteomic approaches to biomarker discovery in prostate and bladder cancers. Proteomics 1: 1264-1270, 2001.

12. Adam B-L, Qu Y, Davis JW, Ward MD, Clements MA and Cazares LH: Serum protein fingerprinting coupled with a pattern-matching algorithm distinguishes prostate cancer from benign prostate hyperplasia and healthy men. Cancer Res 62: 3609-3614, 2002.

13. Fung ET, Weinberger SR, Gavin E and Zhang F: Bioinformatics approaches in clinical proteomics. Expert Rev Proteomics 2: 847-862, 2005.

14. Roboz J: Mass spectrometry in diagnostic oncoproteomics. Cancer Invest 23: 465-478, 2005.

15. Posadas EM, Simpkins F, Liotta LA, MacDonald C and Kohn EC: Proteomic analysis for the early detection and rational treatment of cancer - realistic hope? Ann Oncol 16: 16-22, 2005.

16. Li J, Zhang Z, Rosenzweig J, Wang YY and Chan DW: Proteomics and bioinformatics approaches for identification of serum biomarkers to detect breast cancer. Clin Chem 48: 1296-1304, 2002. 
17. Clarke $\mathrm{CH}$, Buckley JA and Fung ET: proteomics of breast cancer. Clin Chem Lab Med 43: 1314-1320, 2005.

18. Rui Z, Jian-Guo J, Yuan-Peng T, Hai P and Bing-Gen R: Use of serological proteomic methods to find biomarkers associated with breast cancer. Proteomics 3: 433-439, 2003

19. Kim J, Kim SH, Lee SU, et al: Proteome analysis of human liver tumor tissue by two-dimensional gel electrophoresis and matrix assisted laser desorption/ionization-mass spectrometry for identification of disease-related proteins. Electrophoresis 23 . 4142-4156, 2002.

20. Zinkin NT, Grall F, Bhaskar K, et al: Serum proteomics and biomarkers in hepatocellular carcinoma and chronic liver disease. Clin Cancer Res 14: 470-477, 2008.

21. Feng Y, Tian ZM, Wan MX and Zheng ZB: Protein profile of human hepatocarcinoma cell line SMMC-7721: identification and functional analysis. World J Gastroenterol 13: 2608-2614, 2007.

22. Li C, Chen Z, Xiao Z, et al: Comparative proteomics analysis of human lung squamous carcinoma. Biochem Biophys Res Commun 309: 253-260, 2003.

23. Au JS, Cho WC, Yip TT and Law SC: Proteomic approach to biomarker discovery in cancer tissue from lung adenocarcinoma among nonsmoking Chinese women in Hong Kong. Cancer Invest 26: 128-135, 2008

24. Lin JF, Xu J, Tian HY, et al: Identification of candidate prostate cancer biomarkers in prostate needle biopsy specimens using proteomic analysis. Int J Cancer 121: 2596-2605, 2007.
25. Gagnon A, Kim JH, Schorge JO, et al: Use of a combination of approaches to identify and validate relevant tumorassociated antigens and their corresponding autoantibodies in ovarian cancer patients. Clin Cancer Res 14: 764-771, 2008.

26. Petricoin EF and Liotta LA: Proteomic approaches in cancer risk and response assessment. Trends Mol Med 10: 59-64, 2004.

27. Lu HB, Zhou JH, Ma YY, et al: Five serum proteins identified using SELDI-TOF-MS as potential biomarkers of gastric cancer. Jpn J Clin Oncol 40: 336-342, 2010.

28. Anderson NL, Matheson AD and Steiner S: Proteomics: applications in basic and applied biology. Curr Opin Biotechnol 11: 408-412, 2000

29. Ebert MP, Meuer J, Wiemer JC, et al: Identification of gastric cancer patients by serum protein profiling. J Proteome Res 3: 1261-1266, 2004.

30. Liang Y, Fang M, Li J, et al: Serum proteomic patterns for gastric lesions as revealed by SELDI mass spectrometry. Exp Mol Pathol 81: 176-180, 2006.

31. Kozak KR, Amneus MW, Pusey SM, et al: Identification of biomarkers for ovarian cancer using strong anion-exchange ProteinChips: potential use in diagnosis and prognosis. Proc Nat Acad Sci USA 100: 12343-12348, 2003.

32. Diamandis EP: Analysis of serum proteomic patterns for early cancer diagnosis: drawing attention to potential problems. J Natl Cancer Inst 96: 353-356, 2004. 